\title{
Some ruminations on graduate students
}

\author{
Apryll M. Stalcup ${ }^{1}$
}

Published online: 2 August 2016

(C) Springer-Verlag Berlin Heidelberg 2016

After numerous futile attempts by a mentor to engage with a graduate student in preparation for an upcoming presentation, the student presented possibly one of the worst seminars to ever come out of a particular research group. When the mentor attempted to talk to the student about what went wrong in the presentation, the student allegedly told the mentor, "I don't know why it bothers you. My friends liked it!" Sometimes, a student's strong sense of entitlement $[1,2]$ coupled with a lack of appreciation for his/her role in lab reputation and personal responsibility [3] for lab ecology can be exacerbated by the business model of universities that students are consumers of higher education [4].

In the U.S., the minority of students who often give us the most difficulty are sometimes referred to as the "Trophy Generation" [5], children who grew up receiving trophies for just showing up. In China, the "Little Emperors" are thought to be a consequence of the One Child policy [6], and in Sweden, "ouppfostrade" is loosely translated as "badly raised children" [7]. In Ireland, they are called the "Celtic Tiger Cubs," children who grew up during the time of a booming economy. Despite the fact that these students are called different things in such different places, the fact that they are given names suggests that there may be some universality to this phenomenon [5].

Electronic supplementary material The online version of this article (doi:10.1007/s00216-016-9755-x) contains supplementary material, which is available to authorized users.

Apryll M. Stalcup

apryll.stalcup@dcu.ie

1 School of Chemical Sciences, Dublin City University, Glasnevin, Dublin 9, Ireland
Experimentalists often find that the data that does not conform to the model turns out to be the most interesting. So, rather than leaving work frustrated every day, I decided to think of a couple of students who were particularly challenging to mentor as data outliers and used it as an opportunity to do some introspection. This reflection changed my perspective and I offer the following observations. The observations and suggestions below are not intended to be all-inclusive. Rather, this contribution hopes to provoke discussion, both from advisers and students, as a way of inviting further contributions on this topic.

\section{Graduate school as growth}

At its very core, I believe that graduate school is about growth: intellectual, professional, and personal. For me, each student brings his/her own unique skill set, which I visualize as a radar plot (Fig. 1). Part of the delight in cultivating students is the opportunity to discover their unique scientific "radar plot," providing guidance and a safe place for them to be challenged into exploring outside their comfort zones, thereby reinforcing their core strengths as well as alleviating some of their weaknesses. For instance, Student 1 was exceptionally creative and really seemed to understand the theory underpinning the project. However, once it became apparent that the student lacked good practical lab sense or technical skills, the student was transferred to an experimentally more forgiving project, and closer supervision/guidance was provided in the areas of experimental set-up and design. Student 2 was very competent technically but had a weakness in understanding the fundamentals and was challenged to dig in a little deeper into the theoretical underpinnings of the project. 


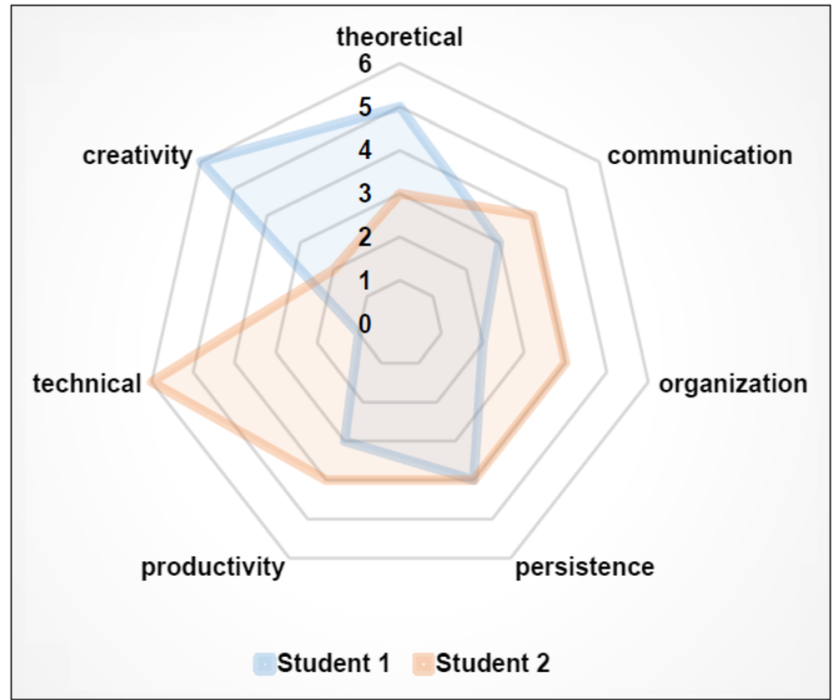

Fig. 1 Example of a student skill set radar plot

Flux in educational models and increasing reliance on metrics in pre-graduate school training have prompted some students to attain an extraordinary ability to non-critically memorize and repeat vast quantities of information. This memorization requires a tremendous amount of effort so they are responding honestly when they say that they have worked really hard. Distinguishing non-critical memorization of "magic words" from actual understanding becomes apparent when results need to be interpreted and additional experiments proposed. Growth is essential here for holistic development.

Early career students may not appreciate that experiments do not always work and that they are not powerful enough to change the laws of chemistry or physics [8]. It is truly a welcome sign of growing scientific maturity when students begin to distinguish bad data from interesting data. This leads to the intellectual ability to recognize that the greatest scientific advances typically arise from the insights gained from experiments that did not work the way he/she thought they should. Indeed, often it is the serendipitous result produced by experiments that experience would strongly guide against that arguably drives a lot of the innovation in academic labs.

Some students do not progress as hoped because they really do not understand, as academics do, that graduate training is supposed to be a semi-structured experience. It is designed to help them develop the skills necessary to become an independent researcher, capable of identifying the next research question to be asked, rationally designing reasonable experiments, and interpreting data (8). Indeed, more than one student has expressed frustration to me that his/her mentor had failed them because their experiments failed. But these students often did not understand that they needed to take ownership of their projects. In this light, calls for normalization of the duration of $\mathrm{PhD}$ projects [9] might need to be tempered to accommodate individual variability in the successful transition to independent researcher.

\section{Learning scientific culture and personal ethics}

Unfortunately, many of the ethical standards and practices that the scientific culture relies on are no longer fundamental to how some societies operate. The increasing call for integration of ethics into the undergraduate and graduate curriculum suggests that some students enter a graduate program without a clear understanding that being a scientist categorically requires absolute honesty about all experimental protocols, all data and analysis. Further, the serendipitous discoveries noted above can be missed entirely if the student does not think beyond "the experiment did not work" or the data is massaged to reflect expected outcomes.

As academics, we understand that socialization of graduate students into the academic and scientific cultures is a critical aspect of mentoring, but we may need to refine our understanding of what that socialization encompasses particularly for first generation students [10] and students from other diverse backgrounds. For example, a student who is exceptionally resistant to suggestions for an appropriate scientific presentation style might be feeling that this guidance is stifling his/her independence and creativity. Further, this very independence likely empowered them to transcend traditional boundaries imposed on them by their socioeconomic background. Initially, this student might have no more idea of what is appropriate in a scientific presentation than I would know how to behave on a film set or at an Irish wake.

Societal incubation intrinsic to some exceptionally challenging or privileged socioeconomic backgrounds can render some very bright students ill-prepared for the customary evaluative discussion at the heart of scientific training and discourse. Hence, critiques of their work, while absolutely necessary, may be misconstrued by these students as either culturally insensitive or irrelevant, no matter how constructively or benevolently offered. It is important to realize that specific problems of, and challenges to, individual graduate students do not map onto a specific ethnic or socioeconomic background. Wildly different backgrounds can produce the same reaction in a student.

A societal predilection for replacing rather than fixing has limited the opportunity for current students to develop handson problem solving abilities. Further, the intervention of "helicopter" or "curling" parents [11] has imbued some of them with a reluctance to engage in solving their own problems. To ascertain a student's experience or aptitude towards problem solving, I started asking students who want to work with me if they have ever changed a flat tire on a car or made a cake from scratch. Their response to this question provides me with some insight as to their potential and willingness to learn 
to troubleshoot and solve their own problems or what sorts of projects to put them on (e.g., theoretical modelling versus experimental).

Some students can be challenging to mentor because they are exceptionally resourceful at circumventing the rules. However, some of these students can turn out quite well if they learn to channel their creativity into productive research pathways.

\section{Mentoring as parenting}

Mentoring graduate students can also be a lot like raising children. I remember Louis C. W. Baker, long-time Head of the Department of Chemistry at Georgetown University, telling in-coming graduate students to talk to other graduate students (in their prospective group) but understand that there comes a point in each graduate student's career when they hate their mentor. Growth is almost always at least a little painful and our students may not always like us while they are going through this process. It is not our job to be our students' friend any more than it is a parent's job to be their child's friend while the child is growing up. That does not mean we can't become friends as they progress in their professional life. Indeed, most academics take great pride in the accomplishments of their former students and look forward to seeing them at professional conferences. Yet, a mentor who worries about being a friend, or being liked, is not going to do the best job for those students that they can.

Most parents know that children can sometimes manipulate poor communication between parents to gain privileges, avoid difficult tasks, or evade consequences. The brighter the individual, the more sophisticated the potential manipulation can be. The same can be said about some students. Graduate students may well be some of the brightest people in their social network. By the time they enter a graduate program, some of them can be quite adept at avoiding tasks that they find onerous or simply don't want to do. Analogous to the dynamics in blended families with parents and stepparents, this aptitude can be particularly problematic in multidisciplinary projects with multiple mentors if communication is poor between the mentors and the students are allowed to drive the narrative. It is only human nature for students to use strategies that worked well for them in the past but these strategies will ultimately not serve them well in their professional life. Hence, all of the student's academic mentors must maintain a respectful, honest, on-going dialogue and transparency between them to deter the student from "gaming" the system or levelling spurious accusations against one or more of their mentors.

As in families, there can also be "sibling rivalry" among research group members with individuals in conflict attempting to engage the mentor in picking sides. Depending upon the situation, the mentor might be well-advised that stepping in like a "helicopter parent" (4) could be denying the individuals in conflict the opportunity to develop experience in compromise that could serve them well in their professional life. It may seem that solving the problem is the primary consideration and that getting the lab back on track is the most important thing to do. Yet, by letting the participants work on the problems, there are likely to be far fewer problems in the future.

\section{Strategies}

So are there some strategies that we can use to be better mentors and guide some of these difficult students but may also have a genuine potential to be good, if not great, scientists? As noted above, these suggestions are not intended to be all-inclusive. Rather, they are intended to provoke discussion and invite further contributions from both advisers and students to the ABCs of Education and Professional Development in Analytical Science.

Some of the issues with graduate students may arise because we have unspoken expectations that may need to be explicitly delineated for this particular generation of students. For instance, it is our expectation that students treat graduate school as a full-time job, at a minimum, but many new graduate students have never held a full-time job.

Some issues with graduate students may arise because they also have unspoken expectations of us. For instance, students who grew up with micromanaged, highly structured childhoods (5) may not be entirely comfortable with the semistructured experience of graduate school. We may need to explicitly tell this generation of students what the mentor's job is and that it does not include following them around to make sure they prepared their solutions correctly. We may need to explain that the mentor's job is to

- identify an interesting problem for the student to work on

- $\quad$ provide the resources and space for the student to do that work safely

- $\quad$ stand back and give the student the opportunity to explore and make the mistakes that would get them fired from a real job

- provide a sounding board by asking the "well-placed questions" to help the student to figure out what went wrong, thereby guiding them towards scientific independence

Because many new graduate students may have limited work experience, we may also need to explain that because the mentor is responsible for providing resources and oversight, the mentor is the Boss.

We may also need to manage some of their expectations (e.g., frequent feedback, flexible hours). Scheduling regular 
one-on-one meetings with each student, separate from group meetings, can help establish some boundaries. Give some structure to the meeting by telling the student to come with answers to the following questions:

- What are your goals (big picture)?

- What have you accomplished in the last unit of time (week, month)?

- What do you propose to accomplish in the next unit of time? Why?

- What are the potential barriers to getting things done?

Make it clear that there are no right or wrong answers to these questions but that answering these questions provide a structure to help them progress on their project, given the general lack of structure in graduate or postdoctoral research. Encourage them to keep a written record of their answers to these questions as a history of their path through graduate school. Insist that the student bring (raw) data that will form the basis of a constructive, evidence-based discussion. Discuss why things did not get done, separating laziness, procrastination, or lack of commitment from genuine lab or scientific problems. Brief notes based on these discussions can provide a written record. In problematic cases, this written record can also be used by the student's thesis or research committee to support a memo to the student describing deficiencies perceived by the committee, guidance on how to address those deficiencies, an anticipated timeline and specific consequences for failure to do so. For laboratory-based projects, the demand for flexible hours may be best addressed by invoking institutional or departmental safety policies.

Further expectations can be conveyed in writing by devising a contract that clearly delineates the roles, responsibilities, and expectations for the student. Indeed, some professional organizations (e.g., Royal Society of Chemistry [12], American Chemical Society [13], American Association for the Advancement of Science [14]) are recommending, and some funding agencies (e.g., U.S. National Institutes of Health, Irish Research Council, Horizon 2020) are now requiring individual development plans (IDP) or personal career development plans (PCDP) for each student. It might be constructive to involve the student in negotiating the terms in the contract in a collaborative process as a way to promote dialogue with the student. Such a contract needs to be clear and to steer between being too detailed (therefore onerous) on one hand, and too vague (therefore unmeasurable) on the other. The contract also needs to be flexible enough to cover students performing research in traditional academic labs as well as the increasing number of students engaged in industrial placements (see Supplemental Material for an example). The student and the mentor could both sign the contract.
Students could be asked to craft a series of essays about their understanding of various aspects of graduate school to engage them in becoming more thoughtful about the process and be asked to discuss these perceptions. This can be in the form of a series of questions such as

- Explain why the student is pursuing an advanced degree in science.

- Discuss their vision of an ideal relationship between a student and a mentor and how the relationship should develop over time.

- Describe the responsibilities the student has towards the mentor, towards the research group, towards the institution, and towards the broader scientific community.

This exercise could be done individually or form part of a departmental orientation or training module for all in-coming students, thereby opening a dialogue or discussion amongst the students under the guidance of a facilitator. Alternatively, students could be provided a survey consisting of a series of research (e.g., literature search) and laboratory ecology (e.g., reagent replacement, laboratory clean-up) statements with scores of 1 to 5 with 1 being $100 \%$ the responsibility of the mentor and 5 being $100 \%$ the responsibility of the student. Again, this could be performed as part of a training module for all in-coming students followed by discussion amongst the students.

Team-building exercises can help alleviate the havoc that some difficult students can wreak in a smooth functioning research group. For instance, assigning group members to organize activities outside the workplace (e.g., group potluck dinner, hiking/hill walking, participation in extramural sports, etc.) or attending conferences together gets everyone out of their comfort zone, while providing them with some shared experiences or memories. One needs to be careful and not have too many of these exercises, however. Recognize that older students, with families, small children, or other responsibilities may have less after-hours flexibility. Group member presentations at conferences can also help unify a group, as members often become protective of one another in the presence of outsiders.

Unfortunately, despite our best intentions, there are some cases in which a student and a mentor simply lack resonance between them. It isn't anyone's fault. As a mentor, realize that neither you nor the student has failed. Departments would be well-served to proactively develop a generic, formalized, transparent pathway for transitioning a student to another mentor before a specific need arises. This promotes respect for both students and mentors and is especially important in the context of increasingly diverse faculty with differences in mentoring styles and diverse students amid growing reports of student incivility [15]. 


\section{Some final thoughts}

Much has been written about the sense of entitlement of the Millennial Generation students $[4,5,15]$ that will eventually replace the Baby Boomer generation [1]. However, there is evidence that some of the less desirable traits associated with the Millennial Generation (e.g., narcissism) are not uniformly distributed across disciplines [16], which could be good news for chemistry. Further, the Millennial Generation's desire for meaningful work, greater workplace diversity, and better work/life balance are very understandable, given that this generation witnessed 9/11, a global economic meltdown with massive numbers of foreclosures, as well as the decline in general earning potential and job security across a wide range of sectors, including academia [17]. Maybe we should cut them some slack - while maintaining standards and expectations. One day, I asked Milton Orchin, an Organic Professor at University of Cincinnati who worked well into his 90s, how they survived the social upheaval of the 1960s. He looked at me with a twinkle in his eye and said "We just stayed true to the science." Ultimately, the future is in the hands of these students and we, as mentors, parents, and leaders, must do our best to authentically prepare them for the many challenges ahead.

Acknowledgments I would like to gratefully acknowledge support from and helpful discussions with Rebecca German (Northeastern Ohio Medical University), Vickie McKee and Andrew Kellett (Dublin City University), Julie Stenken (University of Arkansas), Purnendu (Sandy) Dasgupta (University of Texas at Arlington), Susan Lunte (University of Kansas), and Joel Shulman (University of Cincinnati). The Personal Career Development Plan (PCDP) in the Electronic Supplemental Material was provided by the Marie Skłodowska-Curie Innovative Training Network (ITN) ClickGene (H2020-MSCA-ITN-2014 -c642023). I would also like to acknowledge Dustin Stalcup, who first taught me about parenting, and all of the students with whom I have had the privilege of working over the course of my career - especially the ones alluded to at the beginning of this article.

\section{References}

1. Ng ESW, Schweitzer L, Lyons S. New generation, great expectations: a field study of the millennial generation. J Bus Psychol. 2010;25(2):281-92.

2. Lessard J, Greenberger E, Chen C, Farruggia S. Are youths' feelings of entitlement always "bad"?: evidence for a distinction between exploitive and non-exploitive dimensions of entitlement. $\mathrm{J}$ Adolesc. 2011;34(3):521-9.

3. Fisk GM. "I want it all and I want it now!" An examination of the etiology, expression, and escalation of excessive employee entitlement. Hum Resour Manag Rev. 2010;20(2):102-14.

4. Cain J, Romanelli F, Smith KM (2012) Academic entitlement in pharmacy education. Am J Pharm Edu 76(10):Article189

5. Alsop R. The trophy kids grow up: how the millennial generation is shaking up the workplace. San Francisco: Jossey-Bass; 2008.
6. Crowell T, Hsieh D. Little emperors: is China's one-child policy creating a society of brats? Asiaweek. 1995;21(48):44-50.

7. Hansegard J. Wall Street Journal [Internet]. Brussels, Belgium: Dow Jones Publishing Co. Europe. Available at: http://online.wsj. com/news/articles/SB 10001424052702303519404579 354801246309702. Accessed 10 Feb 2014.

8. Schwartz MA. The importance of stupidity in scientific research. J Cell Sci. 2008;121(11):1771.

9. Salzer R. Educating tomorrow's chemists. Anal Bioanal Chem. 2014;406(14):3251-5.

10. Pain E. Breaking the Class Ceiling Washington, DC: AAAS; 2014. Available at: http://sciencecareers.sciencemag.org/career magazine/previous issues/articles/2014_05_22/caredit.a1400123. Accessed $10 \mathrm{Jul} 2014$.

11. Essig L. Love, Inc [Internet]. Psychology Today 2014. Available at: https://www.psychologytoday.com/blog/love-inc/201404/curlingparents-colbert-and-the-politics-hurt-feelings. Accessed 16 Jun 2016.

12. Royal Society of Chemistry. Available at: http://www.rsc. org/careers/cpd/\#manage-prof-development. Accessed 14 Jul 2016.

13. Krone D, Wenzel TJ. ChemIDP: mapping your career. C\&EN. 2016;94(21):36.

14. American Association for the Advancement of Science. Available at: http://myidp.sciencecareers.org/. Accessed 21 April 2016.

15. Burke LA, Karl K, Peluchette J, Evans WR. Student incivility: a domain review. J Manag Ed. 2014;38(2):160-91.

16. Westerman JW, Bergman JZ, Bergman SM, Daly JP (2011) Are universities creating millennial narcissistic employees? An empirical examination of narcissism in business students and its implications. J Manag Ed

17. Knox J. Permatemps: ghosts of academic present and future. Phi Kappa Phi forum. 2004;84(4):15-7.

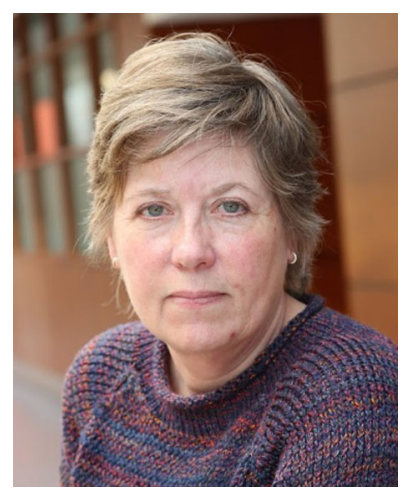

Apryll Stalcup is currently Professor of Chemical Sciences at Dublin City University in Ireland. Her research interests emphasize chromatographic and electrophoretic separation mechanisms, linear solvation energy relationships, exploring new separation methods, and characterizing complex carbohydrates. Her group pioneered the use of sulfated- $\beta$-cyclodextrin, heparin, and quinine as chiral additives in capillary electrophoresis, and demonstrated the wide range of separation modes (reversed phase, normal phase, ion exchange, and ion exclusion) obtainable with surface-confined ionic liquids (SCIL) liquid chromatographic stationary phases. Prof. Stalcup is the author of over 100 publications, reviews, book chapters, and one patent. She is a Fellow of the Institute of Chemistry of Ireland and the Royal Society of Chemistry, a member of the American Chemical Society, the American Association of the Advancement of Science, Sigma Xi, the University of Cincinnati Graduate Fellows, and a Charter member of the University of Cincinnati Chapter's National Academy of Inventors. She was the recipient of the 2015 American Microchemical Society A. A. Benedetti-Pichler Award and the 2011 Cincinnati Section of the American Chemical Society Cincinnati Chemist of the Year Award. She serves as Co-Chair (with Professor Jeremy Glennon, University College Cork) of the 31st International Symposium on Chromatography in 2016. 